\title{
EFFETS DE L'ADMINISTRATION CHRONIQUE DE STÉROÏDES ANTICONCEPTIONNELS SUR L'ÂGE BIOLOGIQUE DES GYCLES OVARIENS DE LA RATTE APRES ARRET DU TRAITEMENT
}

\author{
P. ASCHHEIM \\ Unité de Recherches Gérontologiques, I. N. S. E. R. M., \\ 29 rue Wilhem, \\ 75016 Paris
}

RÉSUMÉ

Après administration orale à des rattes de $4-5$ mois d'âge, pendant 4 ou 8 mois, d'œstrogène, d'un mélange progestagène-eestrogène, mais non de progestagène seul, on obtient un retard net du vieillissement de l'axe hypothalamo-hypophyso-ovarien, se manifestant par une augmentation du nombre de femelles cycliques, une diminution de l'âge biologique de ces cycles (mesuré par un test de vieillissement basé sur l'augmentation avec l'âge, de la sensibilité hypothalamohypophysaire à l'œstrogène capable de déclencher une sécrétion de prolactine) et une diminution du nombre d'hypophyses tumorales ou hypertrophiées à l'autopsie. Le retard du vieillissement par rapport aux témoins se maintient au moins pendant quatre mois après l'arrêt de traitement: cependant, durant cette période, ces rattes vieillissent à leur tour. Le phénomène peut s'expliquer par une " désensibilisation " centrale à l'œestrogène durant le traitement qui est évidemment à l'opposé de l'évolution naturelle des rattes cycliques avec l'âge, à savoir l'augmentation de cette sensibilité à l'ostrogène. La mise au repos de l'ovaire pendant le traitement n'est pas un préalable à l'obtention du retard de vieillissement.

Ce travail a été entrepris pour les raisons suivantes :

a) On dispose de peu de données sur la récupération de la fonction ovarienne et sa régulation chez la Ratte après un traitement chronique par les stéroïđes anticonceptionnels. LIPSCHUTz et al. (I963) indiquent, chez la Souris, que pendant les 8 mois qui suivent un traitement de 3-6 mois, la fécondité est égale à celle de témoins d'âge. Il en est de même après un traitement de 18 mois ; la fécondité est alors basse, les animaux étant séniles. De plus, une proportion notable de souris traitées développent dans l'ovaire des tumeurs de la granulosa, y compris parmi celles qui sont restées fertiles. ZEILMAKER (I969) signale qu'après l'arrêt d'un traitement de 6 mois par le lyndiol, 7 rattes sur 9 redeviennent cycliques et le demeurent pendant 44 mois, alors qu'après l'arrêt d'un traitement de I4 mois, 7 rattes sur 9 passent en cestrus permanent. 
b) La modification de la sensibilité hypothalamo-hypophysaire à l'œstrogène est l'expression majeure du vieillissement de l'axe hypothalamo-hypophyso-ovarien (AschHerm, I970). I'augmentation de cette sensibilité avec l'âge chez la Ratte cyclique peut être utilisée pour la mesure de l'âge biologique des cycles ovariens et ostraux (AschHEIM, I972).

c) Un traitement chronique par des stéroïdes anticonceptionnels réalise une imprégnation chronique des sites stéroïdo-sensibles de l'hypothalamus (et de 1'hypophyse) quel que soit par ailleurs le mécanisme contraceptif mis en jeu. Un test de vieillissement pratiqué après l'arrêt du traitement risque de mieux cerner l'adaptation fonctionnelle obtenue et le mode d'action que la simple observation de la reprise de la cyclicité.

\section{MATÉRIEL, ET MÉTHODES}

Deux substances cestrogènes (éthinylestradiol : LL, et mcstranol : M), deux progestatifs (gestaclon : $\mathrm{G}$, et lynestrénol : L) et deux mélanges progestagène-ostrogène $(\mathrm{G}+\mathrm{EE}, \mathrm{L}+\mathrm{M})$ ont été administrés, incorporés dans les bouchons alimentaires $\mathrm{AO}_{3}$ de la Maison U. A. R.

La concentration est de Io $\mu \mathrm{g} / 20 \mathrm{~g}$ de bouchons pour EE et $\mathrm{M}$; I mg/2o g de bouchons pour $\mathrm{G}$ et $\mathrm{L}$; cependant dans le mélange tout préparé $\mathrm{L}+\mathrm{M}$ (lyndiol), le pourcentage commercial a été respecté : I $\mathrm{mg}$ de $\mathrm{L}$ et $30 \mu \mathrm{g}$ de $\mathrm{M}$ pour $20 \mathrm{~g}$ de nourriture.

Quatre expérjences principales ont été effectuées : 2 de 4 mois et 2 de 8 mois de traitement. Chaque expérience comporte environ 80 rattes au départ : 20 témoins, 20 rattes soumises à l'œestrogène, 20 soumises au progestatif, 20 au mélange. Il y a 4 à 5 rattes par cage. Flles sont àgées de 4 mois au départ, sauf pour la première expérience Schering où elles ont 5 mois d'âge.

Les animaux sont suivis par des pesées régulières, des contrôles de la prise alimentaire, par frottis vaginaux et par une exploration ovarienne, échelonnée au cours des trois derniers mois des traitements courts, systématique avant la fin des traitements longs.

Après arrêt du traitement et reprise des cycles, un premier test de vieillissement est effectué au $4^{\text {e }}$ ostrus, un deuxième test 4 mois plus tard. Un $3^{\mathrm{e}}$ test a été fait 4 mois après le second chez le groupe traité pendant 4 mois par les substances Organon.

Le test de vieillissement consiste en l'injection sous-cutanée, lors de l'ostrus, de $5 \mu g$ de benzoate d'œestradiol. Une réponse " jeune " est fournie far le maintien du cycle ostral ou son allongement de I à 3 jours. Une réponse "âgéc n consiste en une pseudogestation ou, exceptionnellement, un oestrus persistant pour io à zo jours. Le diagnostic de la séquence expérimentale est fait biologiquement : évolution du frottis vaginal, du foids corporel, inspection de l'ovaire. Après cette séquence, les cycles oestraux repronnent normalement. I.incidence des réfonses jeunes et âgées est comparée statistiquement (par table de contingence deux à deux) entre les groupes.

Les rattes sont sacrifiées quelques semaines après le dernier test pour examen du foids et de l'histologie d'organes sélcctionnés.

Dans une expérience supplémentaire, nous avons effectué et comyaré 2 tests de vieillissement, avant et après un traitement de 3 mois par EE et $\mathrm{G}+\mathrm{EE}$.

Dans une autre enfin, des rattes sans traitement stérö̈dien sont amenées, par restriction alimentaire, à un amaigrissement correspondant à celui subi par des rattes soumises à l'oestrogène. puis testées, comme ces dernières, quelque temps après l'arrêt de la rcstriction alimentaire.

Environ $45^{\circ}$ animaux de souche Wistar ont été utilisés.

\section{RÉSULTATS}

\section{A. - Pendant le traitement}

Poids corporel. Toutes les hormones (sauf le gestaclon, progestatif pur) et leurs mélanges font maigrir les rattes immédiatement (perte de poids de IO-I $5 \mathrm{p}$. IOO en 8 -I 5 jours). Le poids se stabilise ensuite, puis augmente lentement. Il ne rejoint le 
poids de départ qu'après 4 mois de traitement, parfois plus tard encore. Après 8 mois de traitement, il ne dépasse le poids initial que de $20-30 \mathrm{~g}$ ou $6-9 \mathrm{~g}$ dans ces groupes contre $89 \mathrm{~g}$ et $73 \mathrm{~g}$ pour les témoins. Les rattes sous gestaclon, par contre, augmentent de poids davantage que les témoins. Après arrêt des traitements, les poids corporels subissent une évolution inverse.

I,es pertes pondérales s'expliquent par la diminution bien connue de la prise alimentaire sous oestrogène, réduite à $50 \mathrm{p}$. Ioo de la normale (qui est de $20 \mathrm{~g} / \mathrm{rat}$ / jour) pendant le premier mois, à $66 \mathrm{p}$. I00, puis $75 \mathrm{p}$. Ioo par la suite. L'augmentation du poids après un progestatif pur $(\mathrm{G})$ est également classique. Il est à noter que 1'association à ce progestatif d'un œestrogène $(\mathrm{G}+\mathrm{EF})$ ou la seule activité œstrogène de l'autre progestatif $\left(L_{1}\right)$ conduisent à une perte de poids ; de ce point de vue, l'ostrogène domine le progestagène.

Les frottis vaginaux indiquent un diœstrus pour les groupes $\mathrm{G}$ et $\mathrm{G}+\mathrm{F} \mathbf{E}$, une stimulation œstrogène qui augmente avec la durée du traitement pour les groupes $\mathrm{L}$ et $\mathrm{L}+\mathrm{M}$. L'exploration ovarienne montre une très grande homogénéité de ces groupes, à la fois pour les animaux d'un même groupe au même moment que tout au long des traitements pour les mêmes animaux : les ovaires sont très petits, sans corps jaunes dans $90 \mathrm{p}$. Ioo des cas, avec seulement de petits follicules visibles. Cela semble traduire un blocage hypophysaire de FSH et LH (l'interstitielle ovarienne est fortement déficiente) réalisé par le progestatif pur seul (gestaclon). L'activité astrogène intrinsèque du progestatif $\mathrm{L}$ ne modifie pas cet état de repos ovarien, (mais explique 1'ostrus vaginal) ; les associations $\mathrm{G}+\mathrm{EI}$, $\mathrm{L}+\mathrm{M}$ non plus. Mais $\mathrm{G}+\mathrm{FE}$ maintient un dicestrus, $\mathrm{I}_{+}+\mathrm{M}$ provoque l'œestrus.

Par contre, les traitements oestrogènes purs : EE et $M$, n'ont pas les mêmes effets sur tous les animaux d'un même groupe au même moment, ni tout au long du traitement sur les mêmes animaux. L,e frottis vaginal révèle, soit des séquences cycliques, soit des séquences de pseudogestation ou des périodes d'œestrus persistant et tous les passages d'un type vers les autres ont été observés. L'exploration ovarienne montre de gros corps jaunes en cas de pseudogestation, des ovaires sans corps jaunes mais avec de grands follicules en cas d'œstrus persistant et, chose curieuse, de gros corps jaunes dans les ovaires des rattes présentant des cycles vaginaux (mais à l'autopsie en postoestrus de quelques-unes de ces rattes " cycliques ", nous n'avons compté que I-2 œufs tubaires). En tout cas, aucun de ces aspects n'évoque un ovaire au repos, mais tous suggèrent plutôt l'existence d'une certaine activité hypophysaire et ovarienne.

\section{B. -- Après l'arrêt du traitement}

Pour l'étude des paramètres essentiels : la reprise des cycles ovariens après arrêt du traitement et leur âge biologique (tabl. I et 2), il convient de noter que les œestrogènes (E) ont le même effet, qu'ils soient d'origine Schering (EE) ou Organon (M) ; les progestagènes $(P)$ ont le même effet, qu'il s'agisse de G (Schering) ou de $\mathrm{I}_{1}$ (Organon), alors que le premier est un progestatif pur, le second conservant une activité œstrogène intrinsèque; $P+F$ ont le même effet (sauf un groupe aberrant) alors que les concentrations respectives en $P$ et $F$ sont légèrement différentes selon la provenance des stéroïdes. Ces résultats sont donc cumulables pour les hormones homologues. 


\section{'ABIIAU I}

Durée des traitemonts : 1 mois

Test : $5 \mu \mathrm{g}$ de benzoate d'oestradiol

\begin{tabular}{|c|c|c|c|c|c|c|}
\hline & \multicolumn{3}{|c|}{1 cr test } & \multicolumn{3}{|c|}{20 test } \\
\hline & $\begin{array}{c}\text { âge } \\
\text { en mois }\end{array}$ & $\begin{array}{c}\begin{array}{c}\text { rattes } \\
\text { cycliques }\end{array} \\
\begin{array}{c}\text { population } \\
\text { totale }\end{array}\end{array}$ & $\begin{array}{c}\text { réponses } \\
\text { jeunes }\end{array}$ & $\begin{array}{c}\text { âge } \\
\text { en mois }\end{array}$ & $\frac{\begin{array}{c}\text { rattes } \\
\text { cycliques }\end{array}}{\begin{array}{c}\text { population } \\
\text { totale }\end{array}}$ & $\begin{array}{c}\begin{array}{c}\text { réponses } \\
\text { jeunes }\end{array} \\
\text { total des } \\
\text { pycliques } \\
\text { testées }\end{array}$ \\
\hline Témoins & $91 / 2$ & $9 / 16$ & $1 / 8$ & 13 & $4 / 16$ & {$[1 / 4]$} \\
\hline $\begin{array}{c}\text { Progestatif } \\
\text { (P) }\end{array}$ & $91 / 2$ & $19 / 19$ & $10 / 19$ & 13 & $11 / 19$ & $1 / 10$ \\
\hline $\begin{array}{l}\text { (listrogène } \\
\text { (E) }\end{array}$ & $91 / 2$ & $16 / 18$ & $12 / 16$ & 13 & $9 / 16$ & $1 / 8$ \\
\hline $\mathrm{P}+\mathrm{E}$ & $91 / 2$ & $18 / 18$ & $15 / 18$ & 13 & $11 / 17$ & $5 / 11$ \\
\hline
\end{tabular}

P : Gestaclon.

E : Ethinylestradiol.

TABLEAU 2

Duvée des traitements : 8 mois

Test : $5 \mu \mathrm{g}$ de benzoate d'oestradiol

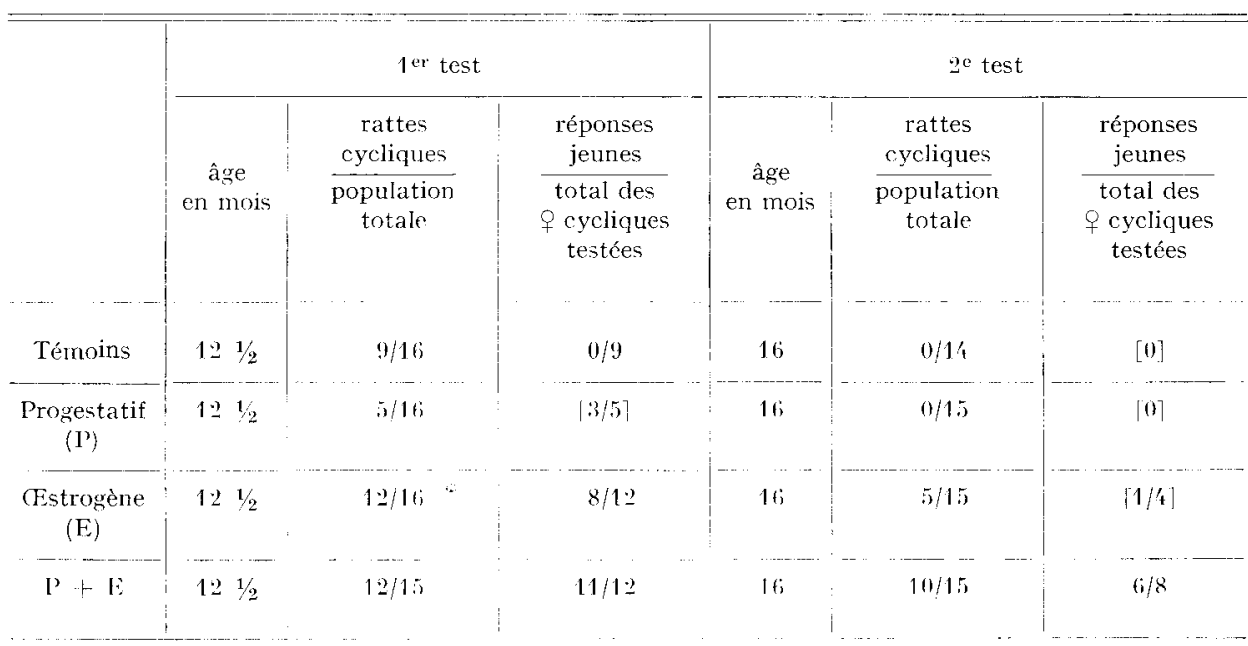

P : Gestaclon.

E : Ethinylestradiol 
De plus, l'efficacité des traitements par $\mathrm{E}$ et $\mathrm{P}+\mathrm{E}$ est similaire et, là encore, pour résumer, nous cumulerons les résultats obtenus :

\section{- Traitement par E et $P+E$ (tab1. 3).}

I. Le pourcentage de femelles cycliques est toujours plus élevé chez les rats traités que chez les témoins : 93,2 p. Ioo contre 70,6 p. Ioo après l'arrêt du traitement de 4 mois (à 9 mois d'âge environ) ; 67,2 p. Ioo contre 32,3 p. IOO 4 mois plus tard ; $70,6 \mathrm{p}$. IOo contre $54,5 \mathrm{p}$. IOO après l'arrêt du traitement de 8 mois (à I2 mois $\mathrm{I} / 2$ d'âge) ; 42,6 p. Ioo contre 3,6 p. Ioo 4 mois plus tard. La différence n'est statistiquement significative que dans ce dernier cas.

TABLEAU 3

Traitements $[\mathrm{Tt}]=E$ plus $P+E$

\begin{tabular}{|c|c|c|c|c|c|c|c|c|}
\hline & \multicolumn{2}{|c|}{$\begin{array}{c}1 \text { mois de 'l't } \\
81 / 2-91 / 2 \\
\text { mois d'âge }\end{array}$} & \multicolumn{2}{|c|}{$\begin{array}{l}\text { 1 mois plus tard } \\
13 \text { mois d'âge }\end{array}$} & \multicolumn{2}{|c|}{$\begin{array}{c}8 \text { mois de } \mathrm{Tt} \\
121 / 2 \text { mois d'âge }\end{array}$} & \multicolumn{2}{|c|}{$\begin{array}{l}\text { 't mois plus tard } \\
16-17 \text { mois d'âge }\end{array}$} \\
\hline & témoins & $\mathrm{Tt}$ & témoins & $\Gamma t$ & témoins & $\mathrm{Tt}$ & témoins & $\mathrm{T} t$ \\
\hline rattes cycliques & $2,1 / 314$ & $68 / 73$ & $10 / 31$ & $43 / 6.1$ & $18 / 33$ & $48 / 68$ & $1 / 28$ & $\begin{array}{l}20 / 47^{*} \\
21 / 64^{* *}\end{array}$ \\
\hline population totale & $70,6 \%$ & $93,2 \%$ & $32,3 \%$ & $67,2 \%$ & $54,5 \%$ & $70,6 \%$ & $3,6 \%$ & $\begin{array}{l}42,6 \% * \\
32,8 \% * *\end{array}$ \\
\hline réponses jeunes & $4 / 23$ & $40 / 5.3$ & $|3 / 10|$ & $23 / 1$ & $3 / 18$ & $37 / 48$ & {$[0]$} & $9 / 17$ \\
\hline $\begin{array}{c}\text { total des } \\
\text { y cyc testées }\end{array}$ & $17, \prime 1 \%$ & $75,5 \%$ & & $56,1 \%$ & $16,7 \%$ & $77,1 \%$ & & $52,9 \%$ \\
\hline
\end{tabular}

Traitement $=P$

\begin{tabular}{|c|c|c|c|c|c|c|c|c|}
\hline & T'émoins & Traitées & Témoins: & Traitées & Témoins & Traitées & Témoins & Traitées \\
\hline- & $\begin{array}{ll}\cdots & -\end{array}$ & i- & - & $\cdots$ & $\cdots$ & -- & & $-\cdots$ \\
\hline rattes cycliques & $2 / 4 / 3 / 4$ & $3 / 36$ & $10 / 31$ & $26 / 37$ & $18 / 33$ & $10 / 33$ & $1 / 28$ & $1 / 31$ \\
\hline population totale & $70,6 \%$ & $89, ; \%$ & $32,3 \%$ & $70,3 \%$ & $51,5 \%$ & $30,3 \%$ & $3,6 \%$ & $3, \geq 1 \%$ \\
\hline $\begin{array}{l}\text { réponses jeunes } \\
\text { total des } \\
\text { t cyc. testées }\end{array}$ & $\begin{array}{c}1 / 2.3 \\
17,4 \%\end{array}$ & $\begin{array}{l}16 / 1 \% \\
47,1 \%\end{array}$ & {$[3 / 10]$} & $\begin{array}{c}5 / 25 \\
\square 0,0 \%\end{array}$ & $\begin{array}{c}3 / 18 \\
16,7 \%\end{array}$ & $|6 / 10|$ & {$[0 \mid$} & $\mid 0 / 1]$ \\
\hline
\end{tabular}

* Sans ou ** avec un groupe aberrant.

2. Le pourcentage de réponses " jeunes" au test de vieillissement est toujours significativement plus élevé chez les rats traités que chez les témoins : 75,5 p. Ioo contre $I 7,4$ p. Ioo après l'arrêt du traitement de 4 mois; 56 ,I p. Ioo contre rien 
4 mois plus tard (seule une minorité de témoins, 10/3I, est encore cyclique, et comptabiliser le résultat de leur test me paraît illusoire); 77, I p. Ioo contre I6,7 p. Ioo après l'arrêt du traitement de 8 mois ; 52,9 p. roo contre rien 4 mois plus tard. Les stéroïdes étant administrés à partir de 4-5 mois d'âge, la similitude des chiffres obtenus après traitement court ou long est frappante. Ce qui semble compter ici, ce n'est pas 1'âge chronologique des animaux, ni la durée des traitements, mais le temps qui sépare le test de l'arrêt des traitements.

75 p. Ioo de réponses " jeunes » avec la dose-test utilisée (5 $\mu \mathrm{g}$ de benzoate d'œstradiol), c'est le pourcentage qu'on rencontre chez des témoins d'environ 4 mois, $55 \mathrm{p}$. Ioo à l'âge de $6-7$ mois environ.

A la reprise des cycles, il y a donc retard manifeste du vieillissement des groupes traités par $\mathrm{E}$ ou $\mathrm{P}+\mathrm{E}$; mais le traitement arrêté, ces groupes vieillissent à leur tour ( $2^{\mathrm{e}}$ test) : $1113^{\mathrm{e}}$ test effectué à l'âge de $\mathrm{I} 7$ mois sur les rattes soumises entre 4 et 8 mois d'âge au traitement Organon le confirme d'ailleurs. Mais, faute de tests échelonnés suffisamment fréquents, nous ne pouvons nous prononcer sur la vitesse à laquelle procède ce vieillissement retardé.

Une donnée supplémentaire est fournie par la comparaison de tests effectués avant et après 3 mois de traitement par $\mathrm{E}$ et $\mathrm{P}+\mathrm{E}$ (Schering). Sur $\mathrm{r} 2$ rattes ayant fourni un test " âgé " juste avant 4 mois d'âge (sur 66 , ce qui est une proportion normale pour un test à $5 \mu \mathrm{g}$ de benzoate d'œstradiol à cet âge), 8 se retrouvent, après le traitement, biologiquement "jeunes " (réponse inversée). Ceci pose le problème d'un "rajeunissement " temporaire. Une $2^{\mathrm{e}}$ expérience de ce type, chez des rattes plus âgées, est en cours pour faire effectuer, à certaines d'entre elles, 2 fois le même " parcours du vieillissement " marqué par le changement de sensibilité à l'œestrogène.

Le retard du vieillissement dî̀ au traitement n'est pas imputable à la restriction alimentaire et à l'amaigrissement consécutif que ce traitement entraîne pendant son application et qui se résorbe après son arrêt. Fn effet, après une période de restriction alimentaire sans administration hormonale, mais comportant pendant la même durée la même consommation alimentaire journalière et entraînant les mêmes changements de poids, le test de vieillissement fournit exactement les mêmes résultats que chez les témoins non restreints.

Les cycles œestraux des rattes ayant été traitées semblent biologiquement normaux (longueur des cycles, nombre d'œufs pondus), même à un âge où les groupes témoins ne comportent, eux, plus guère d'individus cycliques (p. e. à I6 mois I/2 pour les groupes traités entre 4 mois $\mathrm{I} / 2$ et $\mathrm{I} 2$ mois $\mathrm{I} / 2$ d'âge et leurs témoins).

\section{- Traitement par $P$ (tabl. 3).}

I. Le pourcentage de femelles cycliques est plus élevé, mais non significativement, chez des rattes traitées pendant 4 mois, que chez les témoins $(89,5 \mathrm{p}$. Ioo contre $70,6 \mathrm{p}$. IOo) et encore 4 mois plus tard (70,3 p. Ioo contre $32,3 \mathrm{p}$. IOo). Il n'en va pas de même après un traitement de 8 mois $(30,3$ p. Ioo de cycliques chez les traitées, 54,5 p. Ioo chez les témoins), ni 4 mois plus tard.

2. Le pourcentage de réponses " jeunes » au test de vieillissement est plus élevé, mais non significativement, chez les rattes traitées pendant 4 mois que chez les témoins $(47, \mathrm{I}$ p. Ioo contre I7,4 p. IOO). Quatre mois plus tard, cette différence a disparu. P n'a donc pas les mêmes effets que $\mathrm{F}$ et $\mathrm{P}$... Fi. 


\section{- Poids hypophysaires.}

I,ors de l'autopsie, quelque temps après le dernier test, soit à I4 mois, soit à I6-I 8 mois, le fait le plus frappant se rapporte aux poids hypophysaires. En assimilant, sur la base de notre expérience antérieure (AschHEIM et PASTEELS, I963), les hypophyses de plus de $23 \mathrm{mg}$ à des glandes hypertrophiées, voire tumorales (à cellules à prolactine), nous constatons (tabl. 4) que:

- nos traitements ne conduisent pas à la formation d'hypophyses lourdes ; les quantités de stéroïdes administrées ici ne sont donc pas tumorigènes (comme p.e. 20-3o $\mu \mathrm{g}$ d'éthinyl-estradiol par jour pendant un an d'après les indications bibliographiques) ni sous traitement, ni après son arrêt jusqu'à I6-I 8 mois ;

-- au contraire, le pourcentage d'hypophyses hypertrophiées est significativement moindre chez les traités que chez les témoins ( $24 \mathrm{p}$. Ioo contre $48 \mathrm{p}$. Ioo portant sur I8o cas à I6-I 8 mois) ;

- chez les témoins, le nombre de grosses hypophyses augmente de I 2 p. Ioo à 48 p. Ioo entre I4 et $\mathrm{I} 6$ mois. Les traitements par les stéroïdes s'arrêtent, soit à 8-9 mois, soit à I 2 mois. L'effet " protecteur " des stéroïdes est donc aussi un effet " retard " qui, dans les cas extrêmes, reste efficace pendant 9 mois.

TABLEAU 4

Hypophyses d'un poids $\geqslant a ̀$ à $23,0 \mathrm{mg}$

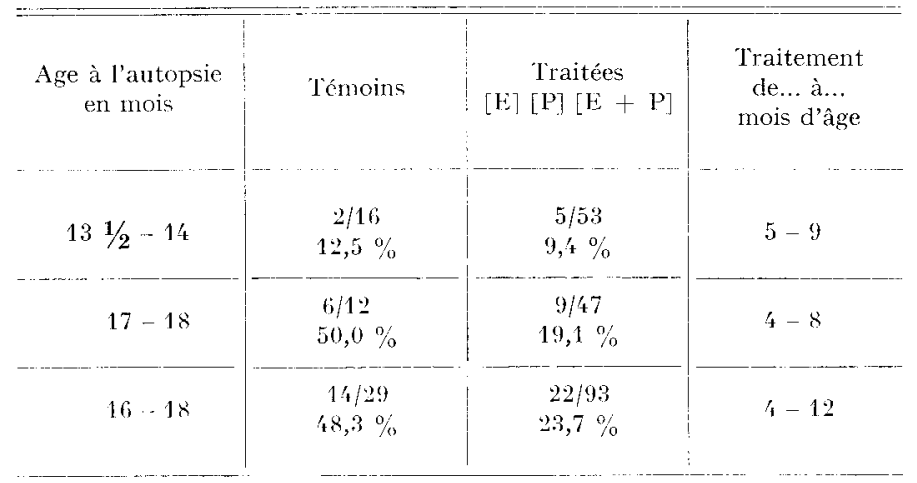

Sur 70 rattes cycliques autopsiées (témoins et traitćes), 3 seulement présentent des hypophyses d'un poids $\geqslant 23,0 \mathrm{mg}$.

Ces constatations rejoignent celles de ZEILMAKER (I969) : aucune hypophyse hémorragique ou hypertrophiée chez 9 rattes traitées pendant I4 mois par le lyndiol, 4-6 mois après son arrêt.

L'histologie des ovaires et du tractus génital sera rapportée ultérieurement. 


\section{DISCUSSION}

L,es traitements $\mathrm{E}$ i et $\mathrm{P}+\mathrm{E}$ (mais non le traitement $\mathrm{P}$ ) conduisent à un retard de vieillissement sur 3 plans : I) le nombre de rattes cycliques est plus grand que chez les témoins ; 2) les cycles eux-mêmes sont biologiquement plus jeunes que chez les témoins ; 3) à l'autopsie, les hypophyses sont beaucoup plus rarement tumorales ou hypertrophiées. Les stéroïdes administrés : I) agissent continuellement sur les sites stéroïdo-sensibles de l'hypothalamus et de 1'hypophyse ; 2) peuvent bloquer l'activité FSH et LH de l'hypophyse et mettre ainsi l'ovaire au repos ; 3) pourraient agir directement sur l'ovaire (ce qui n'entre pas dans le cadre de cette étude, mais jouera nécessairement dans l'interprétation des modifications histologiques de l'ovaire qui existent et vont dans le sens de ce qui a été décrit par LIPSCHUTz et al. (I967) et MyRHE (I972) chez la Souris).

L'atteinte centrale est réalisée par les 3 traitements. Le blocage des gonadotropines est réalisé par $\mathrm{P}$ et $\mathrm{P}+\mathrm{E}$, mais non par $\mathrm{E}$. Le retard du vieillissement est réalisé avec $\mathrm{E}$ et $\mathrm{P}+\mathrm{E}$, non avec $\mathrm{P}$. Le blocage des gonadotropines (par $\mathrm{P}$ ) et le repos ovarien consécutif n'est donc ni indispensable, ni suffisant pour obtenir les résultats sur le vieillissement. C'est l'œestrogène qui est l'hormone nécessaire à cet égard.

Comment agit-elle? Par imprégnation continue des récepteurs neuro-hormonaux sensibles à l'œstrogène situés dans 1'hypothalamus, peut-être aussi par action sur 1'hypophyse. Cette imprégnation continue entraînerait une " désensibilisation " centrale. L'hypothalamus, après l'arrêt du traitement, serait alors devenu moins sensible à l'hormone ovarienne endogène produite après le rétablissement des cycles ou à l'hormone exogène injectée lors du test de vieillissement. Cette notion de désensibilisation est ancienne (HOHLWEG, I934) : chez un Rat mâle, on réalise une inhibition centrale par l'œestrogène ou l'androgène, ce qui conduit à l'arrêt de la spermatogenèse, mais au bout de 7 mois et malgré la poursuite du traitement, il y a reprise de la spermatogenèse. L'effet-rebond découle du même mécanisme. LAKSIIMAN et al. (Ig63) signalent d'ailleurs un tel effet sur le nombre d'ovulations, 50 jours après l'arrêt d'un traitement anticonceptionnel de Ioo jours chez la Ratte.

Dans notre cas, la désensibilisation est suggérée par la reprise par paliers (au bout d'un mois, puis de 2 mois et de 4 mois) de la consommation alimentaire et du poids corporel, paramètres qui restent toutefois inférieurs à ceux des témoins pendant toute la durée du traitement.

Dans l'hypothèse de la désensibilisation à l'œstrogène selon HorrwEG, le retard du vieillissement serait dû à un procédé qui réalise exactement l'inverse du processus naturel de sénescence chez la Ratte cyclique, qui se caractérise par l'augmentation de la sensibilité centrale à l'œestrogène.

Sans vouloir transposer chez la Femme les résultats observés chez la Ratte, ni assimiler doses et mode d'administration des stéroïdes, il est permis de dire que le retard du vieillissement est obtenu après un traitement œstrogène, out progestagèneœstrogène qui correspondrait en gros à une contraception humaine de Io ou de 20 ans, durées qui, actuellement, ne sont pas encore atteintes pleinement chez la Femme soumise depuis le début de l'âge adulte aux stéroïdes contraceptifs. 


\title{
REMERCIEMENTS
}

Travail aiclé par le contrat D. G. R. S. T. $7^{2-7-0056}$ et le contrat I. N. S. E. R. M. - ATP $72-4-4$ I $2-7$.

Nous remercions les laboratoires Schering qui nous ont aimablement fourni l'éthinylestradiol et le gestaclon et les laboratoires Organon qui nous ont fourni le mestranol et le lynestrenol.

\section{SUMMARY}

\author{
THE EFFECTS OF CHRONIC TREATMENT WITH CONTRACEPTIVE STEROIDS \\ ON THE BIOLOGICAL, AGE OF OVARIAN CYCIES \\ IN THE RAT AFTER THE CESSATION OF TREATMENT
}

After oral administration of estrogen, of a progestogen-estrogen mixture, but not of progestogen alone to female rats of $4-5$ months of age, during 4 or 8 months, an obvious delay of the senescence of the hypothalamic-hypophyseal ovarian axis is observed. It is demonstrated by the increase in the number of cyclic females, by the decrease of the biological age of these cycles (measured by an aging-test based on the increasing hypothalamic-hypophyseal sensitivity with age to an injection of estrogen triggering the secretion of prolactin) and by the decrease of the number of tumourous or hypertrophied pituitaries at autopsy. The clelay of aging, as compared with controls, is maintained at least for 4 months after the end of treatment; but during this time experimental rats do age. 'The observed fact may be explained by a central "desensitization " to estrogen during treatment which counteracts exactly the natural evolution with age of cyclic rats, the increase of this sensitivity to estrogen. The complete functional inactivation of the ovary cluring treatment is not necessary to achieve delay of aging.

\section{RÉFÉRENCES BIBLIOGRAPHIQUES}

Aschenm P., r97o. La rétroaction ovarienne dans la régulation hypothalanique de la fonction gonadotrope LH de la Ratte sénile. In: J. Benort et C. Kordon: Colloques Nationaux du C. N. R. S., $\mathrm{n}^{\circ} 927$ : Neuroendocrinologie, Paris, C. N. R. S., 363-376.

Aschreim P., r972. Un test biologique de vicillissement du contrôle du cycle nestral de la Ratte. Gen. Comp. Endocr., 18573.

Aschheim P., Pasteels J. L., I963. Titude histophysiologique de la sécrétion de prolactine chez les rattes séniles. C. R. Acad. Sc. Paris, 257, I373-1 375 .

Hohlweg W., r934. Progynon und Sexualzyklus. Med. Mitt. Sichering A. G., 6, 9-1 3.

Lakshmax A. B., Nelson W. O., I963. "Rebound Effect " of Ovulation-inhibiting Steroid in Rats. Nature, 199, 608-609.

Lipschutz A., Iglesias R., SAlinas S., 1963. Further studies on the recovery of fertility in mice after protracted steroid-induced sterility. J. Reprod. Fert., 6, 99-1 13.

I.ipschutz A., Iglesias R., Panaslevich V. I., Salinas S., r967. Granulosa-cell tumours induced in mice by progesterone. birit. J. Cancer, 21, I44-I52.

Lipschutz A., Iglesias R., Panasevich V. I., Salinas S., ig67. Ovarian tumours and other ovarian changes induced in mice by two I9-nor-contraceptives. Brit. J. Cancer, 21, I53-I59.

MYHRE E., r972. Ovarian morphology following long-tcrm treatment with sex hormones and contraceptive steroids in mice. Acta Path. Microbiol. Seand., 80 A, suppl. 233, 67-75.

ZFrLMAKeR ( . H., I969. liffects of prolonged feeding of an ovulation inhibitor (Lyndiol) on ageing of the hypothalamic-ovarian axis and pituitary yland tumorigenesis in rats. J. Endocr., 43, XXI-XXII. 